\title{
Impact of heparan sulphate binding domain of chemokine CCL21 to migration of breast cancer cells
}

\section{Introduction}

Lymph node metastasis constitutes a key event in breast cancer progression. Chemokines are small proteins, which can promote metastatic spread by inducing cancer cell migration and invasion. Chemokine function is dependant upon their binding to both cell surface heparan sulphate (HS) molecules and to their specific receptor. Our group has demonstrated a significant increase in chemokine receptor CCR7 expression in cancerous breast epithelia compared to healthy controls.

\section{$\underline{\operatorname{Aim}}$}

To test the hypothesis that a non-HS binding forms of chemokine CCL21 can disrupt the normal response to CCL21, therefore reducing the metastasis of CCR7-expressing cancer cells.

\section{Methods}

* Synthesis of truncated CCL21 chemokine $(\Delta 98-134 \mathrm{c}$ terminal basic extension) to investigate a possible linkage between chemokine binding capacity and cell activation.

* Test the ability of Wild type (WT) and mutant-CCL21 to stimulate a dose-dependent increase in intracellular-free calcium in PBMC and breast cancer epithelial cells MDAMB-231.

- Determine the ability of CCL21 to stimulate chemotaxis within a concentration gradient and compare the potential of WT and mutant-CCL21 to stimulate the migration of cells across endothelium.

* Determine the potential of mutant CCL21 for therapeutic blockade of the migration of Breast Cancer cells in vivo.

\section{Conclusions}

* There were a significant increase in chemokine recepto CCR7 expression in cancerous breast epithelia compared to healthy controls and in MDA-231 cells compared to primary Breast epithelial cells. Mutant-CCL21 at concentrations 5 and $10 \mathrm{nM}$ showed potential to mobilise $\mathrm{Ca} 2+$ at levels similar to that produced by WT-CCL21.

* Mutant-CCL21 stimulated no increased in transendothelia cell migration of MDA-MB-231 compared to WT-CCL21.

* Mutant CCL21 in xenograft brain tumor models showed a substantial inhibition of tumour growth compared to WTCCL21

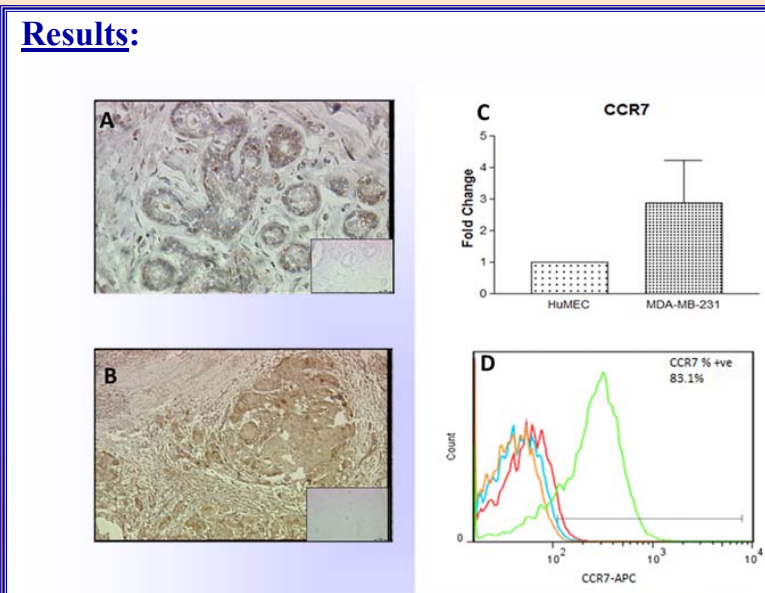

Figure 1: Endogenous expression of CCR7

(A\&B), Immunohistochemical labelling of paraffin embedded healthy (A) and cancerous breast tissue (B) for human CCR7. Inserts show no primary antibody controls. (C), Real time analysis of CCR7 in MDA-MB-231 compared to the expression in HuMEC using GAPDH as a housekeepeer. Data was analysed using the change in expression of each gene according to the increase from the housekeeper gene $(\Delta \mathrm{CT})$. $\mathrm{N}=4$

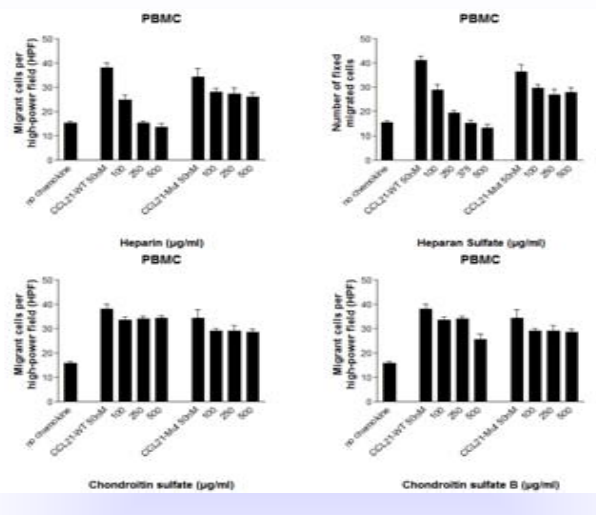

Figure 2: The effect of heparin on chemotactic properties of WT and mutant- CCL21

Inhibition of the chemotactic response of PBMC toward Heparin (A), Heparan sulfate (B), Chondroition sulfate A (C), Chondroition sulfate B (D). $\mathrm{N}=4$
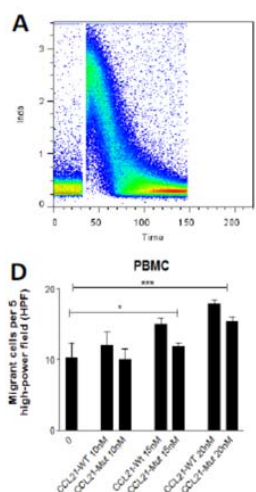

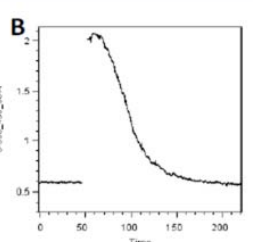

E $\quad 000$

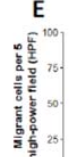

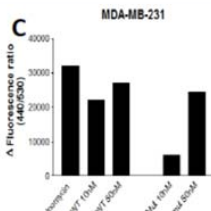

F Transendothelela( PBMc)
Figure 3: Mechanism of action of the non-glycosaminoglycanbinding CCL21 mutants

Calcium flux results: (A\&B), Representative changes in the intracellula $\mathrm{Ca} 2+$ concentration immediately after stimulating of MDA-MB-231 with CCL21 (50nM). (C), Dose response analysis showing changes in the maximal change in intracellular Ca2 + concentration measured in MDA-MB 231 following stimulation with either WT or Mutant CCL21. In vitro chemotaxis experiments: Measurement of PBMC/MDA cell migration across cytokine-activated endothelial cell monolayer grown on filters (transendothelial) (F) or across filter (trans-filter) (D\&E). Invasion across the membrane was assessed after $2 \mathrm{~h}$ for PBMC and $24 \mathrm{~h}$ for MDA-MB-231. $\mathrm{N}=4$

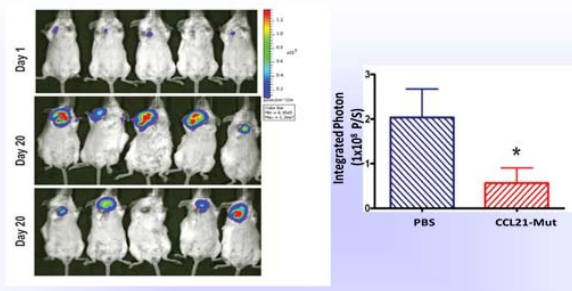

Figure 4: Determine the potential of mutant CCL21 fo therapeutic blockade of the migration of Breast Cancer cells in vivo

Acknowledgment

This work was supported by the internal grant from Qatar University 